\title{
Mechanism of Electro-Optical Switching Hysteresis for (Polymer/Liquid Crystal) Composite Films
}

\author{
Hirotsugu KikUChI, Jun-ichiro NishiwaKi, and Tisato KaJIYAMA* \\ Department of Chemical Science and Technology, Faculty of Engineering, \\ Kyushu University, 6-10-1 Hakozaki, Higashi-ku, Fukuoka 812, Japan
}

(Received September 1, 1995)

\begin{abstract}
The relationship between the hysteresis characteristics of electro-optical switching and the compatibility at the polymer-low molecular weight liquid crystal (LC) interface was studied for the (polymer/LC) composite film prepared by a solvent cast method. The interfacial phase-mixing behavior between polymer and LC components was evaluated on the basis of the dielectric relaxation on an interfacial polarization at the polymer-LC interface and FT-IR ATR spectra. When the polymer was not compatible with LC even in its isotropic state, no hysteresis on the applied voltage-light transmittance relationship was detected for the electro-optical switching. It is suggested that the phase-mixing at the polymer-LC interface induced upon the application of an electric field is a possible mechanism affecting the electro-optical switching hysteresis.
\end{abstract}

KEY WORDS (Polymer/Liquid Crystal) Composite Film / Electro-Optical

Switching Hysteresis / Dielectric Relaxation / Interfacial Polarization / Compatibility /

A novel idea of unconventional compositions of polymers and low molecular weight liquid crystals (LCs) burgeoned in the end of 1970 s in order to apply unique characteristics of LC as a self-supported flexible film. ${ }^{1}$ Extensive studies of the preparation methods, the aggregation structure and the physical properties have been conducted for the composite films consisting of polymer and LC. ${ }^{2-9}$ Recently, (polymer/LC) composite films have attracted much attention because they possess potentially promising applications, for example, large-area displays, switchable windows and light shutters in projection systems. ${ }^{10-19}$ The (polymer/LC) composite film can be reversibly switched between light scattering state and light transmission one upon the removal and the application of an electric field, respectively. The light scattering state and the light transmission one are caused mainly by the random orientation and the unidirectional one of LC directors embedded in the three-dimensional polymer networks, respectively. Particularly, the spatial distortions of LC directors in the order of sub- $\mu \mathrm{m}$ induced by the complicatedly and randomly oriented polymer network assemblies or polymer walls in the composite film are thought to be an important origin for the strong light scattering as well as the mismatch of refractive indices between polymer and LC. ${ }^{17}$ Since the intensity of light scattering is governed by the spatial heterogeneity, the electro-optical characteristics of the (polymer/LC) composite film are strongly dependent on the aggregation state of it. ${ }^{20-22}$ The hysteresis of the electro-optical switching upon an increase process and a decrease one of applied voltage for the (polymer/LC) composite system has been a problem which must be solved for practical application as a display material. However, the detail physical mechanism affecting the electro-

\footnotetext{
* To whom correspondence should be addressed.
} 
optical switching hysteresis is not understood yet.

In this paper, the relationships between the phase-mixing at the polymer-LC interface and the switching hysteresis has been experimentally examined and a possible mechanism of appearance of the switching hysteresis has been discussed.

\section{EXPERIMENTAL}

\section{Materials}

The chemical structures of the constituent polymers and LC materials for the composite films are given in Figure 1. Poly(diisopropyl fumarate) (Pdi-iPF, supplied from Nippon Oil \& Fats Co., Ltd.) and poly(methyl methacrylate) (PMMA, supplied from Mitsubishi Rayon Co., Ltd.) were used as the matrix polymers. E8 (purchased from BDH Chemical Co., Ltd.) and GR-63 (supplied from Chisso Co.) were used as the LC materials. Both E8 and GR-63 are similar nematic mixtures consisting of several cyanophenyl type LCs.

The composite film was prepared by casting the chloroform solution on a glass plate at $308 \mathrm{~K}$. The solution concentration was $3-10$ $\mathrm{wt} \%$. The weight fraction of polymer/LC was $40 / 60$. The film thickness was $15-20 \mu \mathrm{m}$.

\section{Electro-Optical Measurement}

In order to evaluate the electro-optical properties of the composite film, light transmittance changes upon the application of an a.c. electric field were studied by an experimental setup as shown in Figure 2. The composite films were sandwiched between two indium/ tin oxide (ITO) coated glasses. He-Ne laser (wavelength of $632.8 \mathrm{~nm}$, Uniphase) was used as an incident light source. The measurement of the transmitted light intensity through the composite films without any polarizers was carried out with a photodiode under the modulation of an electric field. The transmitted light intensity through the sample cell was normalized by the intensity through a blank cell.

\section{Dielectric Measurement}

The dielectric properties were evaluated by

1) Polymer

a) Poly (diisopropyl fummarate) (Pdi-iPF)

$T_{\mathrm{g}}=500 \mathrm{~K}$ (decomposition)

$n=1.464$

$M_{n}=1.5 \times 10^{5}$

b) Poly(methyl methacrylate) (PMMA)

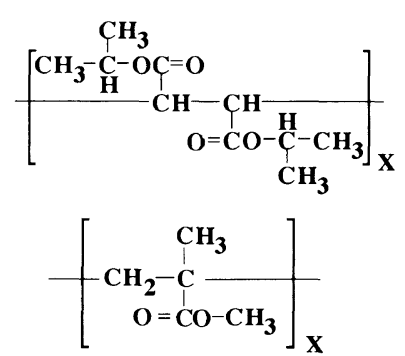

1) Liquid Crystal

$T_{\mathrm{g}}=377 \mathrm{~K}$

$n=1.492$

$M_{n}=1.8-2.0 \times 10^{5}$

a) $\mathrm{E} 8(\mathrm{BDH})$ nematic mixture consisting of cyano-

phenyl LCs

$T_{\mathrm{NI}}=345 \mathrm{~K}$

$n_{\perp}=1.527 \quad n_{\|}=1.774$

$\Delta n=0.247 \quad n_{\mathrm{AVE}}=1.609$

b) GR-63 (Chisso Co., Ltd.)

$T_{\mathrm{NI}}=336.5 \mathrm{~K}$

$\varepsilon_{\perp}=5.0 \quad n_{\perp}=1.522$

$\varepsilon_{\|}=16.8 \quad n_{\|}=1.744$

$\Delta \varepsilon=11.8 \quad \Delta n=0.222$

$\varepsilon_{\mathrm{AVE}}=8.93 \quad n_{\mathrm{AVE}}=1.596$

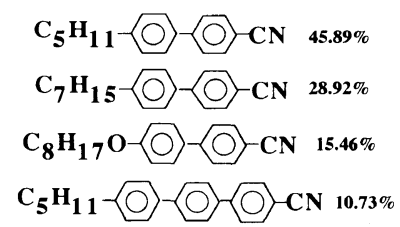

Figure 1. The chemical structures of the constituent polymers and LC materials for the composite films. 


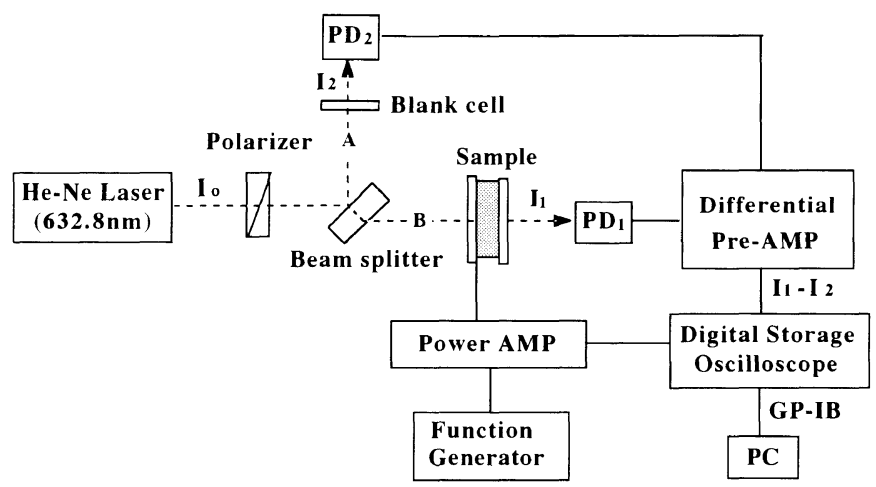

Figure 2. Schematic diagram of the measuring system of electro-optical properties of the composite film.

the time domain method. The transient current of the sample upon the application of a stepwise d.c. voltage from 0 to $1.0 \mathrm{~V}$ generated by a multi-function synthesizer (NF1940) was recorded with a digital memory (Hioki HI-8830). The time-dependent current was converted into the frequency dependence of the complex dielectric constant by the Fourier transform.

\section{FT-IR Measurement}

Infrared spectra were recorded on a Nicolet Model 510 Fourier transform spectrophotometer at a resolution of $2 \mathrm{~cm}^{-1}$. The Spectra-Tech internal reflection attachment was used for ATR measurement. Ge prism (refractive index: 4.0) for the incident angle of $30^{\circ}$ was used as an ATR substrate. In order to evaluate the amount of LC penetration into the polymer by the ATR measurement, the polymer film surface was contacted with the $\mathrm{LC}$ for $2 \mathrm{~h}$ and then, the LC was rinsed with n-hexane (for Pdi-iPF) or methanol (for PMMA).

\section{RESULTS AND DISCUSSION}

\section{Electro-Optical Switching of (Polymer/LC)} Composite Film

Figure 3 shows the applied voltage dependence of the transmittance for the (PdiiPF/E8: 40/60) composite film. The transmittance-voltage curves for processes of an in-

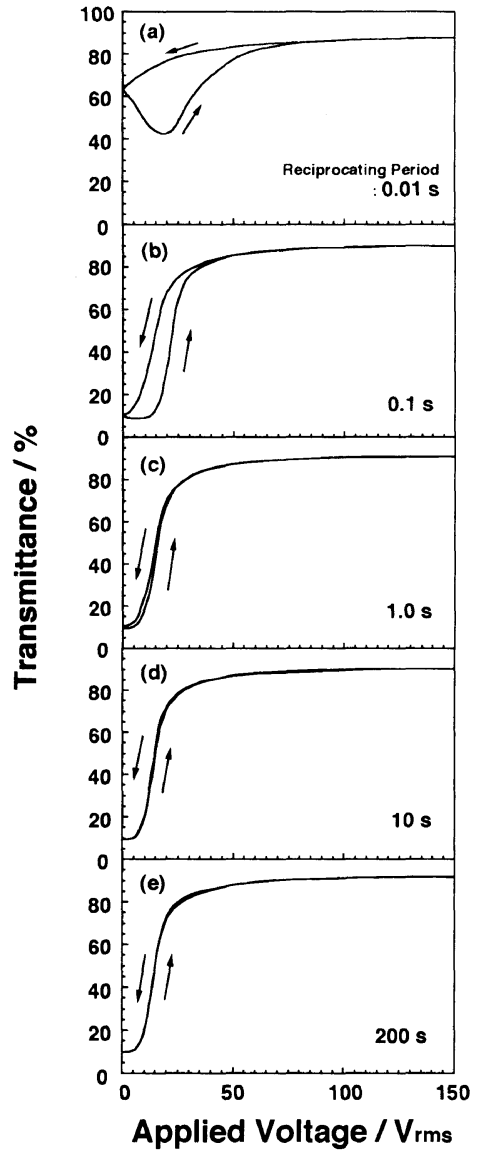

Figure 3. The applied voltage dependence of the transmittance for the (Pdi-iPF/E8: 40/60 wt\%) composite film. 
creasing and a decreasing applied voltage are shown as a function of reciprocating period. The amplitude of an applied voltage was modulated linearly. When the reciprocal period was longer than $1.0 \mathrm{~s}$, the electro-optical hysteresis of the (Pdi-iPF/E8) composite film was hardly observed in the reciprocating process as shown in Figures 3(c)-(e). However, the degree of the hysteresis became more apparent with reducing the reciprocating period, in other word, a higher electro-optical switching frequency. The hystereses in the shorter range of reciprocating period shown in Figures 3(a), (b) seem to be responsible for the retardation of the $\mathrm{LC}$ molecular reorientation upon the amplitude modulation of an applied

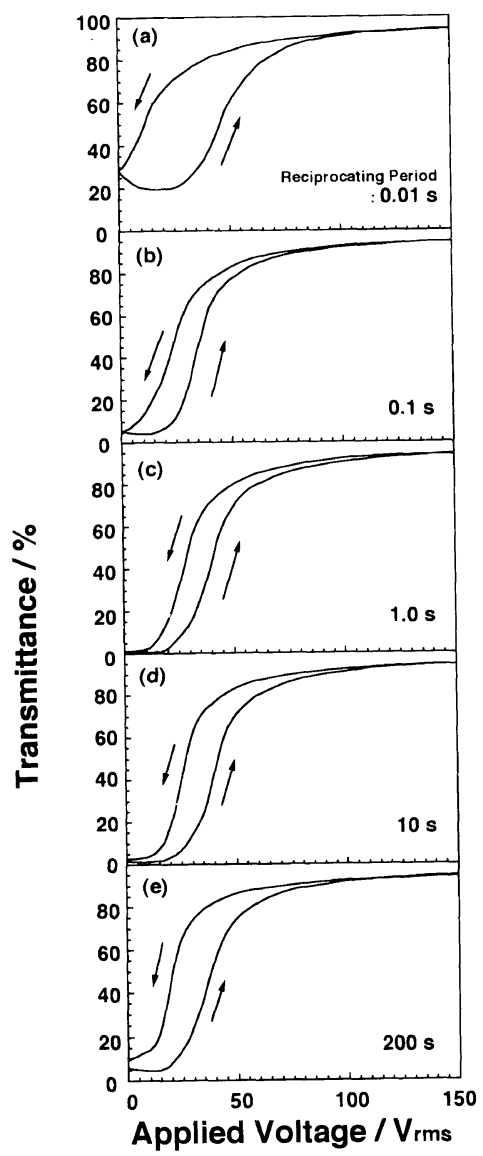

Figure 4. The applied voltage dependence of the transmittance for the (PMMA/E8: 40/60 wt \%) composite film. electric field. Since the decay time of the electro-optical switching for the composite film is in the order of $\mathrm{ms},{ }^{14,17,22}$ the time lag of the electro-optical response can not be negligible in the range of the reciprocating period shorter than $0.1 \mathrm{~s}$. Figure 4 shows the applied voltage dependence of thetransmittance for the (PMMA/E8: 40/60 wt \%) composite film as a function of reciprocating period. More apparent hystereses were observed in all cases of reciprocating period from $0.01 \mathrm{~s}$ to $200 \mathrm{~s}$ in comparison with the results shown in Figure 3 . In the case of the short reciprocating periods of 0.01 and $0.1 \mathrm{~s}$, the hystereses would result from the retardation of the LC molecular reorientation in the same way as the (PdiiPF/E8) composite film. However, the hystereses in the range longer than $1.0 \mathrm{~s}$ are considered to be caused by another mechanism, which will be discussed later.

\section{Dielectric Analysis of Phase-Separated Struc- ture Based on Interfacial Polarization}

Figure 5(a) shows the dielectric composite model of the (polymer/LC) composite film. ${ }^{16,18}$ If an alternating electric field, $E$ is applied to a dielectric material, then an alternating electric displacement, $D$ is detected as a response with a phase different angle, $\delta$. The relationship between $E$ and $D$ is written by using a complex dielectric constant, $\varepsilon^{*}$ as given by eq 1 .

$$
D=\varepsilon^{*} E
$$

and

$$
\begin{aligned}
& \varepsilon^{*}=\varepsilon^{\prime}-i \varepsilon^{\prime \prime} \\
& \frac{\varepsilon^{\prime \prime}}{\varepsilon^{\prime}}=\tan \delta
\end{aligned}
$$

where $\varepsilon^{\prime}$ and $\varepsilon^{\prime \prime}$ are the real and imaginary components of $\varepsilon^{*}$. It has been reported that the dielectric characteristics for the (polymer/LC) composite films can be roughly described on the basis of a series-connected dielectric composite. ${ }^{18}$ In the series model, the magnitude of the electric displacement, $D$ in 
the polymer phase is equal to that in the LC phase as follows.

$$
D=\varepsilon_{\text {COMP }}^{*} E=\varepsilon_{\mathrm{LC}}^{*} E_{\mathrm{LC}}=\varepsilon_{\mathrm{P}}^{*} E_{\mathrm{P}}
$$

where $\varepsilon_{\text {COMP }}^{*}, \varepsilon_{\mathrm{LC}}^{*}, \varepsilon_{\mathrm{P}}^{*}, E_{\mathrm{LC}}$ and $E_{\mathrm{P}}$ are the complex dielectric constants of the dielectric composite, the LC phase, the polymer phase, the electric field in the LC phase and that in the polymer phase, respectively. The magnitude of the total electric field, $E$ is written in the assumption of the additivity law based on each volume fraction, $\phi_{j}$ as given by eq 5 . The subscript $j$ means either LC (liquid crystal) or $\mathrm{P}$ (polymer) phase.

$$
E=\phi_{\mathrm{LC}} E_{\mathrm{LC}}+\phi_{\mathrm{P}} E_{\mathrm{P}}
$$

From eq 4 and 5 , we have

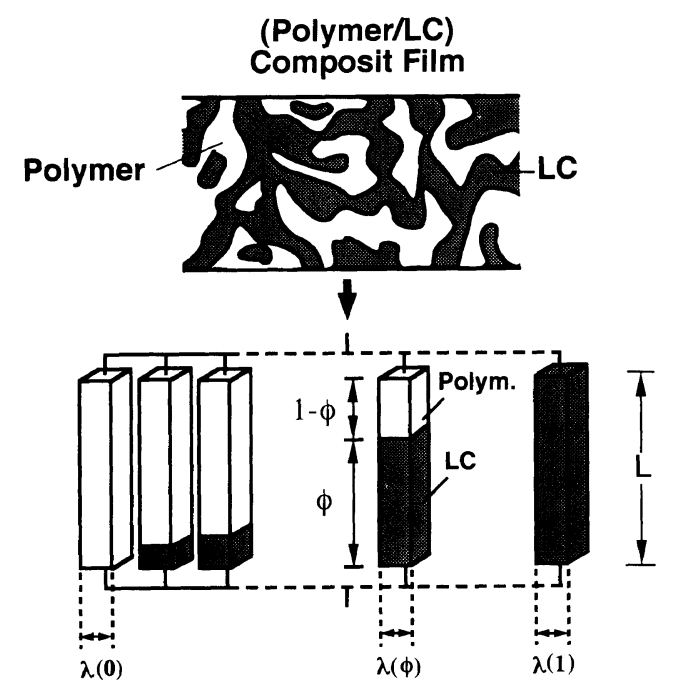

(a)

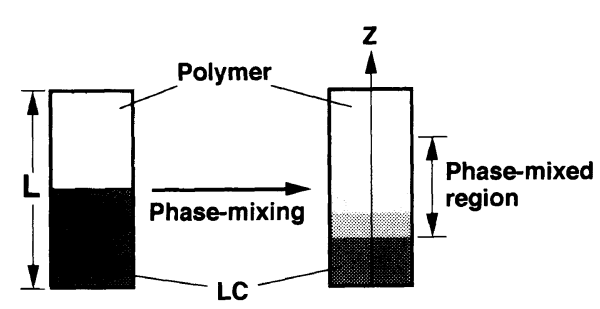

(b)

Figure 5. The dielectric composite model of the (polymer/LC) composite film.

$$
\frac{1}{\varepsilon_{\mathrm{COMP}}^{*}}=\frac{\phi_{\mathrm{LC}}}{\varepsilon_{\mathrm{LC}}^{*}}+\frac{\phi_{\mathrm{P}}}{\varepsilon_{\mathrm{P}}^{*}}
$$

Then, $\varepsilon_{\text {COMP }}^{*}$ is given by eq $7-9$.

$$
\varepsilon_{\mathrm{COMP}}^{*}=\varepsilon_{\mathrm{COMP}}^{\prime}-i \varepsilon_{\mathrm{COMP}}^{*}
$$

where

$$
\varepsilon_{\mathrm{COMP}}^{\prime}=\frac{\phi_{\mathrm{LC}} \varepsilon_{\mathrm{LC}}^{\prime}\left(\varepsilon_{\mathrm{P}}^{\prime 2}+\varepsilon_{\mathrm{P}}^{\prime \prime 2}\right)+\phi_{\mathrm{P}} \varepsilon_{\mathrm{P}}^{\prime}\left(\varepsilon_{\mathrm{LC}}^{\prime 2}+\varepsilon_{\mathrm{LC}}^{\prime \prime 2}\right)}{\left(\phi_{\mathrm{LC}} \varepsilon_{\mathrm{P}}^{\prime}+\phi_{\mathrm{P}} \varepsilon_{\mathrm{LC}}^{\prime}\right)^{2}+\left(\phi_{\mathbf{L C}} \varepsilon_{\mathrm{P}}^{\prime \prime}+\phi_{\mathrm{P}} \varepsilon_{\mathrm{LC}}^{\prime \prime}\right)^{2}}
$$

and

$$
\varepsilon_{\text {COMP }}^{\prime \prime}=\frac{\phi_{\mathrm{LC}} \varepsilon_{\mathrm{LC}}^{\prime \prime}\left(\varepsilon_{\mathrm{P}}^{\prime 2}+\varepsilon_{\mathrm{P}}^{\prime \prime 2}\right)+\phi_{\mathrm{P}} \varepsilon_{\mathrm{P}}^{\prime \prime}\left(\varepsilon_{\mathrm{LC}}^{\prime 2}+\varepsilon_{\mathrm{LC}}^{\prime \prime 2}\right)}{\left(\phi_{\mathrm{LC}} \varepsilon_{\mathrm{P}}^{\prime}+\phi_{\mathrm{P}} \varepsilon_{\mathrm{LC}}^{\prime}\right)^{2}+\left(\phi_{\mathrm{LC}} \varepsilon_{\mathrm{P}}^{\prime \prime}+\phi_{\mathrm{P}} \varepsilon_{\mathrm{LC}}^{\prime \prime}\right)^{2}}
$$

Furthermore, in a frequency range where any relaxation phenomena due to thermal molecular motions are not observed, the relationship between $\varepsilon^{\prime \prime}$ and the electric conductivity, $\sigma$ can be approximated by

$$
\varepsilon_{j}^{\prime \prime}=\frac{\sigma_{j}}{\omega}, \quad(j=\mathrm{P}, \mathrm{LC})
$$

where $\omega$ is an angular frequency. Then, we obtain

$$
\varepsilon_{\mathrm{COMP}}^{*}=\varepsilon_{\infty}-i \frac{\sigma}{\omega}+\frac{\varepsilon_{\mathrm{s}}-\varepsilon_{\infty}}{1+i \omega \tau_{0}}
$$

and

$$
\begin{gathered}
\varepsilon_{\mathrm{COMP}}^{\prime}=\varepsilon_{\infty}+\frac{\varepsilon_{\mathrm{s}}-\varepsilon_{\infty}}{1+\omega^{2} \tau_{0}^{2}} \\
\varepsilon_{\mathrm{COMP}}^{\prime \prime}=\frac{\sigma}{\omega}+\frac{\left(\varepsilon_{\mathrm{s}}-\varepsilon_{\infty}\right) \omega \tau}{1+\omega^{2} \tau_{0}^{2}}
\end{gathered}
$$

where

$$
\begin{gathered}
\varepsilon_{\infty}=\frac{\varepsilon_{\mathrm{LC}}^{\prime} \varepsilon_{\mathrm{P}}^{\prime}}{\phi_{\mathrm{P}} \varepsilon_{\mathrm{LC}}^{\prime}+\phi_{\mathrm{LC}} \varepsilon_{\mathrm{P}}^{\prime}} \\
\varepsilon_{\mathrm{s}}=\frac{\phi_{\mathrm{P}} \varepsilon_{\mathrm{P}}^{\prime} \sigma_{\mathrm{LC}}^{2}+\phi_{\mathrm{LC}} \varepsilon_{\mathrm{LC}}^{\prime} \sigma_{\mathrm{P}}^{2}}{\left(\phi_{\mathrm{P}} \sigma_{\mathrm{LC}}+\phi_{\mathrm{LC}} \sigma_{\mathrm{P}}\right)^{2}} \\
\tau_{0}=\frac{\phi_{\mathrm{P} \varepsilon_{\mathrm{LC}}+\phi_{\mathrm{LC}} \varepsilon_{\mathrm{P}}^{\prime}}}{\phi_{\mathrm{P}} \sigma_{\mathrm{LC}}+\phi_{\mathrm{LC}} \sigma_{\mathrm{P}}}
\end{gathered}
$$




$$
\sigma=\frac{\sigma_{\mathrm{LC}} \sigma_{\mathrm{P}}}{\phi_{\mathrm{P}} \sigma_{\mathrm{LC}}+\phi_{\mathrm{LC}} \sigma_{\mathrm{P}}}
$$

Equation 11 is the analogous expression to the Debye's relaxation form. In the case of Debye's equations, the relaxation time and the relaxation intensity are dependent on both the mobility and the magnitude of dipole moments of constituent molecules. However, the relaxation behavior of dielectric composite is due to an interfacial polarization, which depends on the dielectric and conductive properties of each phase. From eq 14 and 15, the relaxation intensity of the interfacial polarization for the dielectric composite is given by

$\varepsilon_{\mathrm{s}}-\varepsilon_{\infty}=\frac{\phi_{\mathrm{LC}} \phi_{\mathrm{P}}\left(\varepsilon_{\mathrm{LC}}^{\prime} \sigma_{\mathrm{P}}-\varepsilon_{\mathrm{P}}^{\prime} \sigma_{\mathrm{LC}}\right)^{2}}{\left(\phi_{\mathrm{P}} \varepsilon_{\mathrm{LC}}^{\prime}+\phi_{\mathrm{LC}} \varepsilon_{\mathrm{P}}^{\prime}\right)\left(\phi_{\mathrm{P}} \sigma_{\mathrm{LC}}+\phi_{\mathrm{LC}} \sigma_{\mathrm{P}}\right)^{2}}$

Hence, the relaxation behavior originated from the interfacial polarization can be observed when

$$
\varepsilon_{\mathrm{s}}-\varepsilon_{\infty} \neq 0
$$

Then, from eq 18 and 19 ,

$$
\frac{\varepsilon_{\mathrm{LC}}^{\prime}}{\varepsilon_{\mathrm{P}}^{\prime}} \neq \frac{\sigma_{\mathrm{LC}}}{\sigma_{\mathrm{P}}}
$$

In the most cases of the dielectric composites consisting of the LC and the polymer phases, eq 21 can be held because $\sigma_{\mathrm{LC}}$ is much lager than $\sigma_{\mathrm{P}}$ on account of a fluid nature of LC.

$$
\frac{\varepsilon_{\mathrm{LC}}^{\prime}}{\varepsilon_{\mathrm{P}}^{\prime}} \ll \frac{\sigma_{\mathrm{LC}}}{\sigma_{\mathrm{P}}}
$$

Therefore, the relaxation behavior associated with the interfacial polarization can be observed for the almost all (polymer/LC) composites. Equations 4-18 are valid only when both the completely phase-separated LC and polymer phases are simply connected in series. However, if both the phases mix each other at their interface, the expression of $\varepsilon_{\text {Cомp }}^{*}$ should be a continuous function with the position from the interface. Then, a new dielectric model in which all dielectric parameters continuously change along the $z$-axis should be employed as shown in Figure 5(b). Here, the direction of the normal axis to the film surface is taken as the $z$-axis. Then, eq 22 should be used instead of eq 6 .

$$
\frac{1}{\varepsilon_{\text {COMP }}^{*}}=\frac{1}{L} \int_{0}^{L} \frac{1}{\varepsilon^{\prime}(z)-i \varepsilon^{\prime \prime}(z)} \mathrm{d} z
$$

where $\varepsilon^{\prime}(z)$ and $\varepsilon^{\prime \prime}(z)$ are the real and the imaginary components of the complex dielectric constants in a thin region of $\mathrm{d} z$. Then, $\varepsilon_{\text {COMP }}^{\prime}$ and $\varepsilon_{\text {COMP }}^{\prime \prime}$ can be written by eq 23 and 24 , respectively.

$$
\begin{aligned}
& \varepsilon_{\mathrm{COMP}}^{\prime}=\frac{X}{X^{2}+Y^{2}} \\
& \varepsilon_{\mathrm{COMP}}^{\prime \prime}=\frac{Y}{X^{2}+Y^{2}}
\end{aligned}
$$

where

$$
\begin{aligned}
& X=\frac{1}{L} \int_{0}^{L} \frac{\omega^{2} \varepsilon^{\prime}(z)}{\omega^{2} \varepsilon^{\prime}(z)^{2}+\sigma(z)^{2}} \mathrm{~d} z \\
& Y=\frac{1}{L} \int_{0}^{L} \frac{\omega \sigma(z)}{\omega^{2} \varepsilon^{\prime}(z)^{2}+\sigma(z)^{2}} \mathrm{~d} z
\end{aligned}
$$

The relaxation behavior of the dielectric composites with a continuous gradient of the LC and the polymer fractions is strongly related to the spatial distribution state of the magnitudes of dielectric constant, $\varepsilon^{\prime}(z)$ and electric conductivity, $\sigma(z)$ which are the functions of a position along $z$-axis. In the relaxation region of the interfacial polarization, $\varepsilon_{\text {COMP }}^{\prime}$ is found to decrease with frequency from a value of $\varepsilon_{\mathrm{s}}(\omega \rightarrow 0)$ to $\varepsilon_{\infty}(\omega \rightarrow \infty)$. Here, $\varepsilon_{\mathrm{s}}$ and $\varepsilon_{\infty}$ can be given by eq 27 and 28 , respectively.

$$
\varepsilon_{\mathrm{s}}=\lim _{\omega \rightarrow 0} \varepsilon_{\operatorname{COMP}}^{\prime}=\frac{L \int_{0}^{L} \frac{\varepsilon^{\prime}(z)}{\sigma(z)^{2}} \mathrm{~d} z}{\left(\int_{0}^{L} \frac{1}{\sigma(z)} \mathrm{d} z\right)^{2}}
$$




$$
\varepsilon_{\infty}=\lim _{\omega \rightarrow \infty} \varepsilon_{\text {COMP }}^{\prime}=\frac{L}{\int_{0}^{L} \frac{1}{\varepsilon^{\prime}(z)} \mathrm{d} z}
$$

Then, the relaxation intensity of the interfacial polarization for the dielectric composite is expressed by eq 29 .

$\varepsilon_{\mathrm{s}}-\varepsilon_{\infty}=L\left[\frac{\int_{0}^{L} \frac{\varepsilon^{\prime}(z)}{\sigma(z)^{2}} \mathrm{~d} z}{\left(\int_{0}^{L} \frac{1}{\sigma(z)} \mathrm{d} z\right)^{2}}-\frac{1}{\int_{0}^{L} \frac{1}{\varepsilon^{\prime}(z)} \mathrm{d} z}\right]$

From eq 29, the relaxation intensity of the interfacial polarization for the composite film depends on the $z$-profiles of $\varepsilon^{\prime}(z)$ and $\sigma(z)$. When $\varepsilon^{\prime}(z)$ and $\sigma(z)$ are independent of $z$, the relaxation intensity is zero. In other words, when the polymer and LC phases are completely mixed each other, no interfacial polarization occurs. Therefore, the structural change from the phase-separated state into the phase-mixed one in the (polymer/LC) composite film can be detected from a decrease in the relaxation intensity on the interfacial polarization at the polymer-LC interface.

The dielectric relaxation of the composite film was experimentally evaluated by the time domain method. The stepwise electric field from 0 to $E_{0}$ was applied to the sample at time of $0, t=0$. A transient electric current resulted from electric charging flows through the sample. By the Fourier transform of the time dependence of the transient current density, $J(t)$, the frequency dependence of the complex dielectric constant of the sample, $\varepsilon^{*}(\omega)$ can be obtained based on eq 30 .

$$
\varepsilon^{*}(\omega)=\varepsilon_{\infty}+\frac{1}{E_{0}} \int_{0}^{\infty} J(t) \mathrm{e}^{-i \omega t} \mathrm{~d} t-i \frac{\sigma}{\omega}
$$

Figures 6 and 7 show the frequency dependence of the relative dielectric constant, $\left(\varepsilon^{\prime}-\varepsilon_{\infty}\right) / \varepsilon_{0}$ and relative dielectric loss constant, $\varepsilon^{\prime \prime} / \varepsilon_{0}$ for the (Pdi-iPF/GR-63) composite film and (PMMA/GR-63) one, respectively. Here, $\varepsilon_{0}$ is the dielectric constant of vacuum, that is $8.854 \times 10^{-12} \mathrm{~F} \mathrm{~m}^{-1}$. In general, dielectric relaxation phenomena associated with thermal molecular motions of LC appear in a frequency range higher than $10 \mathrm{MHz} .{ }^{23}$ The relaxation phenomenon corresponding to the local mode of PMMA side chain might be observed near a frequency range studied here. However, since the relative relaxation intensity, $\left(\varepsilon_{\mathrm{s}}-\varepsilon_{\infty}\right) / \varepsilon_{0}$, for the local mode is less than $3,{ }^{24}$ it can be distinguished from the interfacial polarization of which relative relaxation intensity can be $30-40$ depending on the $z$-profile of mixing ratio. From the individual dielectric property of polymer and LC, the relaxation frequency for the interfacial polarization can be roughly estimated from eq 16 to be $1-10 \mathrm{~Hz}$ at room temperature (on condition $\phi_{\mathrm{P}} \approx 0.4, \phi_{\mathrm{LC}} \approx$ $0.6, \varepsilon_{\mathrm{P}}^{\prime} / \varepsilon_{0} \approx 3-4, \varepsilon_{\mathrm{LC}}^{\prime} / \varepsilon_{0} \approx 10-20, \sigma_{\mathrm{P}} \approx 10^{-11}-$ $\left.10^{-12} \mathrm{~S} \mathrm{~m}^{-1}, \sigma_{\mathrm{LC}} \approx 10^{-9} \mathrm{~S} \mathrm{~m}^{-1}\right)$. As shown in Figures 6 and 7, the relaxation intensities are about $30-40$ and $8-10$ for the (Pdi-iPF/GR63) and the (PMMA/GR-63) composite films, respectively. Furthermore, the relaxation frequency ranged $1-10 \mathrm{~Hz}$ at $283-293 \mathrm{~K}$. Because of both the reasons (1) the relaxation intensities observed in Figures 6 and 7 are much higher than relaxation intensity associated with a thermal molecular motion in polymers, and (2) the dielectric relaxation frequencies are close to the expected value for the interfacial polarization, it is reasonable to consider that the relaxations shown in Figures 6 and 7 correspond to the interfacial polarizations at polymer and LC in the composite film. The increase in the relaxation frequency with temperature might be due to an increase in the electric conductivity with temperature as expected from eq 16 . The relaxation intensity of the (Pdi-iPF/GR-63) composite film was little affected by the nematic-isotropic phase transition of GR-63. On the other hand, the relaxation intensity of the (PMMA/GR-63) composite film decreased around $323 \mathrm{~K}$, and then, disappeared at $333 \mathrm{~K}$ being just below $T_{\mathrm{NI}}$ of GR-63. The disappearance of the 


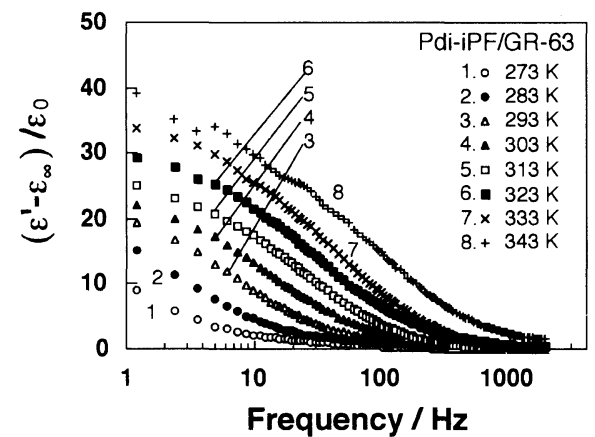

(a)

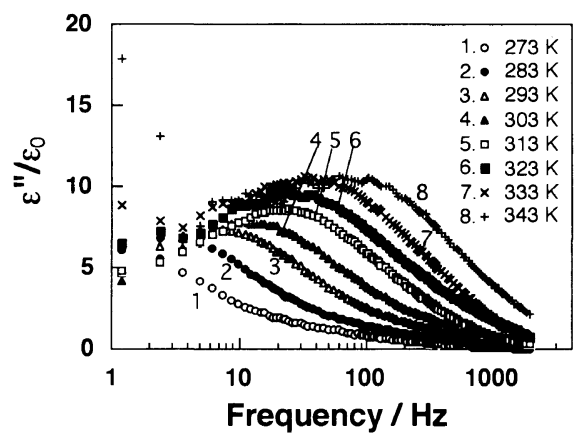

(b)

Figure 6. The frequency dependence of the relative dielectric constant and relative dielectric loss constant for the (Pdi-iPF/GR-63) composite film. $\varepsilon_{0}$ is the dielectric constant of vacuum, that is $8.854 \times 10^{-12} \mathrm{~F} \mathrm{~m}^{-1}$.

interfacial polarization results from the phasemixing of PMMA and GR-63 as theoretically analyzed above. Those results revealed that the clear phase-separated structure in the (PdiiPF/GR-63) composite film was still present even above $T_{\mathrm{NI}}$ of GR-63, however, that in the (PMMA/GR-63) one vanished in the vicinity of $T_{\mathrm{NI}}$ of GR-63. If both the polymer and the LC are mixed each other, the optical heterogeneity in the composite film should reduce, resulting in an increase in the transparency of the composite film. Assuming that the phase-mixing is enhanced by application of an electric filed, the mechanism of the electro-optical switching hysteresis observed for the (PMMA/E8) composite system as shown in Figure 4 could be physically explained as mentioned later.

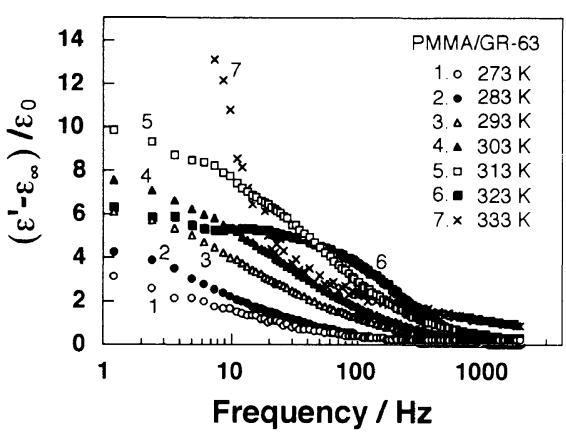

(a)

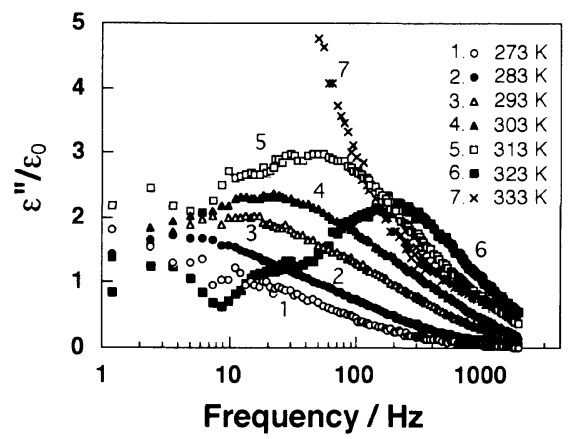

(b)

Figure 7. The frequency dependence of the relative dielectric constant and relative dielectric loss constant for the (PMMA/GR-63) composite film. $\varepsilon_{0}$ is the dielectric constant of vacuum, that is $8.854 \times 10^{-12} \mathrm{~F} \mathrm{~m}^{-1}$.

\section{Electric Field-Induced Phase Mixing at Poly-} mer-LC Interface

Figure 8 shows the FT-IR spectra for (a) PMMA, (b) GR-63 and (c) PMMA after contacting with GR-63. The absorption peak at $1740 \mathrm{~cm}^{-1}$ for PMMA in Figure 8 (b) can be assigned to $\mathrm{C}=\mathrm{O}$ stretching. An absorption band corresponding to $\mathrm{C} \equiv \mathrm{N}$ stretching was observed at $2228 \mathrm{~cm}^{-1}$ in the case of GR-63 as shown in Figure 8 (b). If the GR-63 penetrated into PMMA during contacting together, the $\mathrm{C} \equiv \mathrm{N}$ stretching band at $2228 \mathrm{~cm}^{-1}$ must be detected even after GR-63 was removed with $n$-hexane from the PMMA surface. Therefore, the result in Figure 8 (c) indicates the (PMMA/GR-63) phase-mixing at the interface. The amount of absorbed GR-63 


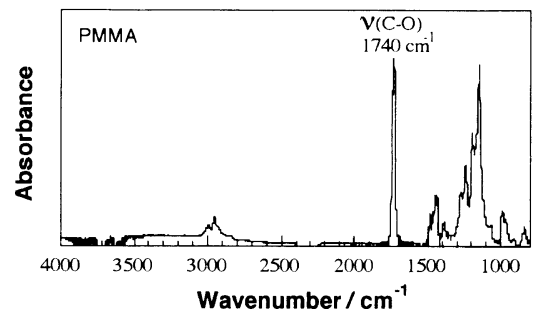

(a)

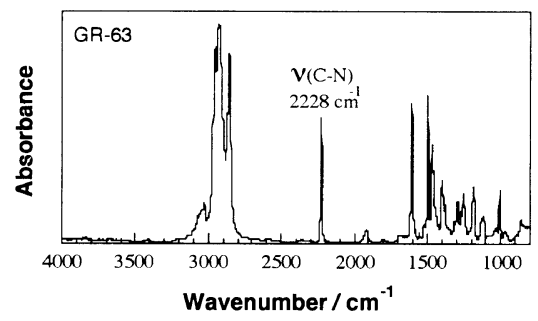

(b)

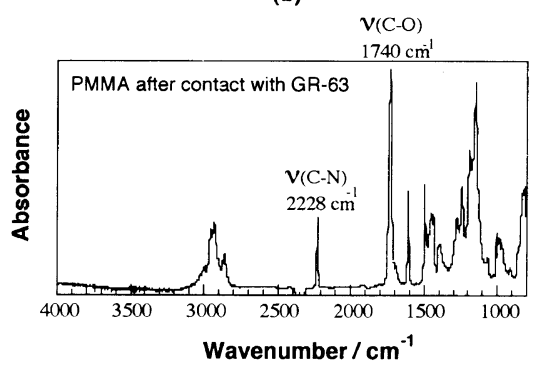

(c)

Figure 8. The FT-IR spectra for (a) PMMA, (b) GR-63 and (c) PMMA after contacting with GR-63.

into PMMA can be evaluated by the relative intensity of $\mathrm{C} \equiv \mathrm{N}$ stretching normalized by that of $\mathrm{C}=\mathrm{O}$ stretching. In the case of the (Pdi-iPF/GR-63) system, the same analysis method can be applied. The comparison of the phase-mixing behavior at the (PMMA/GR-63) and the (Pdi-iPF/GR-63) interface was performed with FT-IR ATR method.

The FT-IR ATR spectra of the $\mathrm{C} \equiv \mathrm{N}$ stretching for the PMMA film surface after contacting with GR-63 are shown in Figure 9(a) as a function of contacting temperature. The analytical depth was estimated to be $0.7 \mu \mathrm{m}$ from the incident angle, $30^{\circ}$. The absorption peaks were normalized by the intensities of $\mathrm{C}=\mathrm{O}$ band of PMMA. The intensity of absorption peak increased with rising tempera-

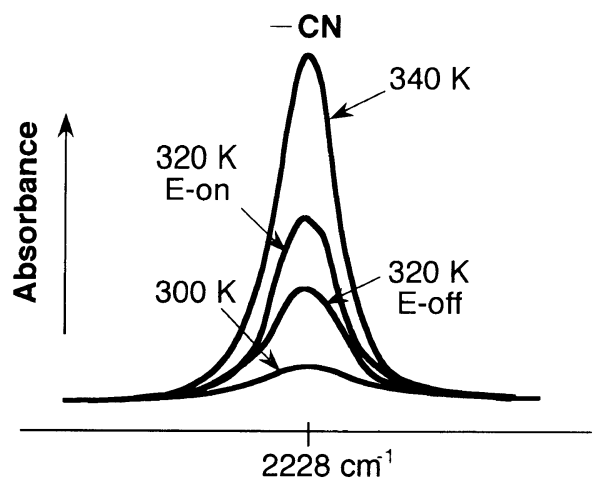

(a)

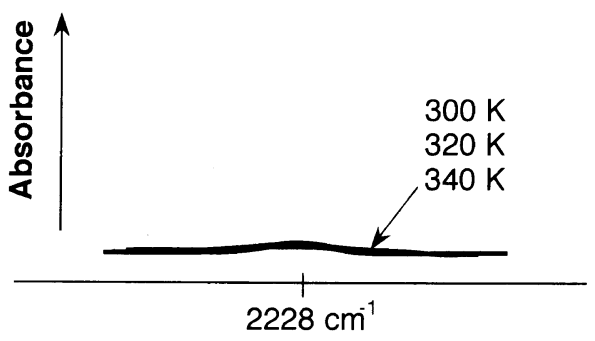

(b)

Figure 9. The FT-IR ATR spectra of the $\mathrm{C} \equiv \mathrm{N}$ stretching for the PMMA film surface after contacting with GR-63.

ture, suggesting that GR-63 penetrated into the contacting PMMA film and the magnitude of penetration increased with temperature. Furthermore, the absorption of GR-63 in PMMA was enhanced by applying an electric filed under isotherm from the comparison of the curves indicated as E-on and E-off in Figure 9(a). This is the first experimental evidence that the compatibility between PMMA and cyanophenyl type LC can be enhanced by applying an electric field. On the other hand, the spectra of Pdi-iPF after contacting with GR-63 were little affected by rising temperature as shown in Figure 9 (b) and also by applying an electric field. It is confirmed by FT-IR ATR analysis that Pdi-iPF and GR-63 are not compatible even higher temperature than $\mathrm{T}_{\mathrm{NI}}$ of GR-63.

Based on the results described above, a possible mechanism of the electro-optical switching hysteresis can be proposed as follows. 
When an a.c. electric field is applied to the (polymer/LC) composite film, dielectric loss energy due to the interfacial polarization and the electric conduction is generated in the composite film. The dielectric loss energy would be converted into heat and/or an endothermic structural change in the composite film. If the polymer used as a matrix is not compatible to LC such as Pdi-iPF to E8 or GR-63, a large portion of the dielectric loss energy is converted into just heat. However, if the polymer is compatible to LC such as PMMA to E8 or GR-63, a part of the dielectric loss energy would be converted into a structural change in the composite film and enhance the miscibility between polymer and LC as suggested by the dielectric relaxation and FT-IR ATR results. An increase in phasemixing of polymer and $\mathrm{LC}$ induces a decrease in the optical heterogeneity, that is, a decrease in the intensity of light scattering in the composite film. If the phase-separated structure is changed, the recovery process to the original structure after removing an electric field should take long time because it needs the molecular diffusion. Therefore, the phase-separated structure in the increasing process of the applied electric voltage in the electro-optical switching is not same as that in the decreasing one, if the polymer and LC become compatible by applying an electric field such as (PMMA/E8 or GR-63) system. That is to say, the light transmittance in the increasing process of the applied electric field is lower than that in the decreasing process, in which polymer and LC may be more phase-mixed, as observed in Figure 4. On the other hand, if the polymer and LC are not compatible such as (Pdi-iPH/E8 or GR-63) system, obvious electro-optical hysteresis in the range of reciprocating period longer than $1.0 \mathrm{~s}$ is not observed as shown in Figure 3.

\section{CONCLUSION}

The compatibility of polymer and LC in the
(polymer/LC) composite film was examined with measurements of the dielectric relaxation of the interfacial polarization arisen between them and the FT-IR ATR performed at their interface. The electro-optical switching hysteresis observed in the increasing and decreasing processes of an applied electric voltage depended on the compatibility between polymer and LC. A possible mechanism that the phase-mixing of polymer and LC induced by application of an electric field would be a main cause of the hysteresis was proposed. As a result, an incompatible combination of polymer and LC is required in order to develop a composite film with no electro-optical switching hysteresis.

\section{REFERENCES}

1. T. Kajiyama, Y. Nagata, E. Maemura, and M. Takayanagi, Chem. Lett., 679 (1979).

2. T. Kajiyama, Y. Nagata, S. Washizu, and $\mathbf{M}$. Takayanagi, J. Membrane Sci., 11, 39 (1982).

3. T. Kajiyama, S. Washizu, and M. Takayanagi, $J$. Appl. Polym. Sci., 29, 3955 (1984).

4. S. Washizu, I. Terada, T. Kajiyama, and $\mathbf{M}$. Takayanagi, Polym. J., 16, 307 (1984).

5. T. Kajiyama, S. Washizu, and Y. Ohmori, $J$. - Membrane Sci., 24, 73 (1985).

6. T. Kajiyama, S. Washizu, A. Kumano, I. Terada, M. Takayanagi, and S. Shinkai, J. Appl. Polym. Sci., Appl. Polym. Symp., 41, 327 (1985).

7. H. Kikuchi, M. Katayose, A. Takahara, S. Shinkai, and T. Kajiyama, Kobunshi Ronbunshu, 43, 669 (1986).

8. H. Kikuchi, A. Kumano, T. Kajiyama, M. Takayanagi, and S. Shinkai, J. Chem. Soc. Jpn., Chem. Ind. Chem., 423 (1987).

9. T. Kajiyama, H. Kikuchi, and S. Shinkai, $J$. Membrane Sci., 36, 243 (1988).

10. H. G. Crighead, J. Cheng, and S. Hackwood, Appl. Phys. Lett., 40, 22 (1982).

11. J. L. Fergason, SID Int. Symp. Dig. Tech., 16, 68 (1985).

12. P. S. Drzaic, J. Appl. Phys., 60, 2142 (1986).

13. J. W. Doane, N. A. Vaz, B. G. Wu, and S. Zumer, Appl. Phys. Lett., 48, 27 (1986).

14. T. Kajiyama, A. Miyamoto, H, Kikuchi, and Y. Morimura, Chem. Lett., 813 (1989).

15. T. Kajiyama, H. Kikuchi, A. Miyamoto, S. Moritomi, and J. C. Hwang, Chem. Lett., 817 (1989).

16. H. Kikuchi, A. Miyamoto, S. Kobayasi, Y. 
Morimura, and T. Kajiyama, J. Chem. Soc. Jpn., Chem. Ind. Chem., 434 (1990).

17. A. Miyamoto, H. Kikuchi, Y. Morimura, and T. Kajiyama, New Polym. Mater., 2, 27 (1990).

18. A. Miyamoto, H. Kikuchi, S. Kobayashi, Y. Morimura, and T. Kajiyama, Macromolecules, 24, 3915(1991).

19. K. Park, H. Kikuchi, and T. Kajiyama, Trans. Mater. Res. Soc. Jpn., 13, 18 (1993).

20. S. Tanioka, H. Kikuchi, and T. Kajiyama, Trans.
Mater. Res. Soc. Jpn., 13, 28 (1993).

21. K. Park, H. Kikuchi, and T. Kajiyama, Polym. J., 26, 895 (1994).

22. H. Kikuchi, F. Usui, and T. Kajiyama, submitted to Polym. J., (1995).

23. D. Lippens, J. P. Parneix, and A. Chapoton, J. Phys., 38, 1465 (1977).

24. Y. Ishida and K. Yamafuji, Kolloid Z., 177, 97 (1961). 